\title{
A WEAK-STAR RATIONAL APPROXIMATION PROBLEM CONNECTED WITH SUBNORMAL OPERATORS
}

\author{
JAMES DUDZIAK
}

(Communicated by John Conway)

\begin{abstract}
Let $\mu$ be a positive Borel measure on a compact subset $K$ of the complex plane. Denote the weak-star closure in $L^{\infty}(\mu)$ of $R(K)$ by $R^{\infty}(K, \mu)$. Given $f \in R^{\infty}(K, \mu)$, denote the weak-star closure in $L^{\infty}(\mu)$ of the algebra generated by $R^{\infty}(K, \mu)$ and the complex conjugate of $f$ by $A^{\infty}(f, \mu)$. This paper determines the structure of $A^{\infty}(f, \mu)$. As a consequence, a solution is obtained to a problem concerned with minimal normal extensions of functions of a subnormal operator.
\end{abstract}

\section{INTRODUCTION}

Let $K$ be a compact subset of the complex plane $\mathbf{C}$ and let $\mu$ be a finite, positive, Borel measure on $K$. Define $R^{\infty}(K, \mu)$ to be the weak-star closure in $L^{\infty}(\mu)$ of $R(K)$. Here $R(K)$ denotes, as usual, the uniform closure in $C(K)$ of the holomorphic rational functions with poles off $K$. Given $f \in R^{\infty}(K, \mu)$, define $A^{\infty}(f, \mu)$ to be the weak-star closure in $L^{\infty}(\mu)$ of the algebra generated by $R^{\infty}(K, \mu)$ and the complex conjugate $\bar{f}$ of $f$. The problem addressed in this paper is that of determining when $A^{\infty}(f, \mu)=L^{\infty}(\mu)$, or more broadly, that of determining the structure of $A^{\infty}(f, \mu)$.

Although this problem is purely function-theoretic, it is equivalent to an operator-theoretic question concerning the minimal normal extension (mne) of a function of a subnormal operator. The equivalence follows. For more details, see $[\mathrm{D}]$.

Theorem. $A^{\infty}(f, \mu)=L^{\infty}(\mu)$ iff mne $f(S)=f($ mne $S)$ for every subnormal operator $S$ whose spectrum is contained in $K$ and whose scalar-valued spectral measure can be taken to be $\mu$.

The problem was first raised in [CO] where it was solved for any polynomially convex $K$. In this case $R^{\infty}(K, \mu)$ reduces to $P^{\infty}(\mu)$, the weak-star closure in $L^{\infty}(\mu)$ of the holomorphic polynomials. Subsequently, the problem was

Received by the editors December 31, 1988.

1980 Mathematics Subject Classification (1985 Revision). Primary 30E10, 41A65; Secondary 47B20. 
solved for any finitely connected (and even some infinitely connected) $K$ in [D]. Recently, in [GRT] the problem has been solved for arbitrary $K$ by clever elementary means while in $[\mathrm{C}]$ a new proof of the $P^{\infty}$ result of $[\mathrm{CO}]$ has been set forth whose novel ingredient is the use of disintegration of measures. The present paper uses the disintegration technique of $[C]$ to recapture and improve upon the result of [GRT]. Although the resulting proof is no longer elementary, it is rather elegant.

\section{Preliminaries concerning $R^{\infty}(K, \mu)$}

For the duration of this section, $K, \mu$, and $f$ shall be fixed as in the introduction. The elementary theory of $R(K)$, as can be found in the second chapter of [G1], for example, shall be assumed known. It is necessary to expand the definition of $R(K)$ somewhat. Given a Borel subset $\Delta$ of $K$, define $R(\Delta)$ to be the uniform closure in $C(K)$ of the set of functions $\Phi$ of the form

$$
\Phi(z)=\int \frac{\varphi(\zeta)}{\zeta-z} d A(\zeta)
$$

where $\varphi$ varies over all compactly supported, bounded Borel functions on $\mathbf{C}$ that vanish on $\Delta$. Here $A$ denotes area measure on $\mathbf{C}$. Given a complex measure $\sigma$ on $K$, let $\hat{\sigma}\left(z_{0}\right)=\int \frac{d \sigma(z)}{z-z_{0}}$ be the Cauchy transform of $\sigma$. An elementary and useful fact which follows from Fubini's Theorem is that $\sigma$ annihilates $R(\Delta)$ iff $\hat{\sigma}=0 A$-a.e. off $\Delta$. Thus for $\Delta=K$, the newly defined $R(\Delta)$ is indeed the old $R(K)$. Also $R\left(\Delta_{1}\right) \subseteq R\left(\Delta_{2}\right)$ whenever $\Delta_{2} \subseteq \Delta_{1} \subseteq K$.

The other facts needed concerning $R(\Delta)$ are not so easily seen and followed.

Proposition. (a) $R(\Delta)$ is a uniform algebra on $K[\mathrm{G} 2,3.2]$.

(b) If $g \in R(\Delta)$ extends to be holomorphic in a neighborhood of $z_{0} \in K$, then

$$
\frac{g(z)-g\left(z_{0}\right)}{z-z_{0}} \in R(\Delta)
$$

[G2, 4.6 and 4.7].

(c) Given $z_{0} \in K$, the functions in $R(\Delta)$ which extend to be holomorphic near $z_{0}$ are uniformly dense in $R(\Delta)$ [G2, 4.6 and 4.8].

Define the envelope $E$ of $\mu$ with respect to $K$ to be the set of all $z \in K$ that possess a complex representing measure $\mu_{z}$ for $R(K)$ such that $\mu_{z}<<\mu$ and $\mu_{z}(\{z\})=0$. For each fixed $z \in E$, the complex homomorphism of evaluation at $z$ on $R(K)$ extends uniquely to a weak-star continuous, complex homomorphism on $R^{\infty}(K, \mu)$. This extension is given by the functional $g \in$ $R^{\infty}(K, \mu) \mapsto \breve{g}(z) \in \mathbf{C}$ where $\check{g}(z)=\int g d \mu_{z}$. Unfixing $z \in E$, one has a point function $\breve{\mathrm{g}}: E \mapsto \mathrm{C}$ associated with each measure function $g \in R^{\infty}(K, \mu)$. Furthermore, $\check{g}_{n} \rightarrow \check{g}$ pointwise boundedly on $E$ whenever $g_{n} \rightarrow g$ boundedly $\mu$-a.e. in $R^{\infty}(K, \mu)$.

The other facts needed concerning these notions are not so easily seen and follow. 
Proposition. (d) $E$ is a Borel set [Ch, X.5].

(e) $K^{\prime}(E) \subseteq R^{\infty}(K, \mu)$ [Ch, X.6].

(f) For any $g \in R(E), \check{g}=g \mid E$ [Ch, X.6].

(g) Given $g \in R^{\infty}(K, \mu)$, there exists a sequence $\left\{g_{n}\right\}_{n>1}$ from $R(E)$ such that $\left\|g_{n}\right\|_{K} \leq\|g\|_{\mu}$ and $g_{n} \rightarrow g$ pointwise $\mu$-a.e. [Ch, XI.10].

From all this, one can see that $\check{g}: E \mapsto \mathbf{C}$ is a Borel function for $g \in$ $R^{\infty}(K, \mu)$.

Finally, given a Borel subset $\Delta$ of $K$ and a finite, positive, Borel measure $\sigma$ on $K$, define $R^{\infty}(\Delta, \sigma)$ to be the weak-star closure in $L^{\infty}(\sigma)$ of $R(\Delta)$. The characterization of annihilating measures mentioned earlier yields a HartogsRosenthal Theorem for $R(\Delta)$, to wit, $R(\Delta)=C(K)$ whenever $A(\Delta)=0$. The following is then immediate.

Proposition. (h) $R^{\infty}(\Delta, \sigma)=L^{\infty}(\sigma)$ whenever $A(\Delta)=0$.

\section{PRELIMINARIES CONCERNING THE DisintegRATION OF MEASURES}

Let $X$ be a locally compact, separable, metric space and let $\mu$ be a finite, positive, Borel measure on $X$. As opposed to $L^{\infty}(\mu)$ and $L^{1}(\mu)$, which are Banach spaces of equivalence classes of $\mu$-measurable functions on $X$, it will also be necessary to consider $\mathscr{L}^{\infty}(\mu)$ and $\mathscr{L}^{1}(\mu)$, which are the corresponding pseudo-normed spaces of Borel functions on $X$. Fix $\varphi \in \mathscr{L}^{\infty}(\mu)$. Denote the $\mu$-essential range of $\varphi$ by $Y$ and consider the finite, positive, Borel measure $\nu$ on $Y$ defined by $\nu(\Delta)=\mu\left(\varphi^{-1}(\Delta)\right)$. Designate the set of Borel probability measures on $X$ by $\mathscr{P}(X)$.

An assignment $\zeta \in Y \mapsto \lambda_{\zeta} \in \mathscr{P}(X)$ is called a disintegration of $\mu$ with respect to $\varphi$ iff for every $\psi \in \mathscr{L}^{1}(\mu)$ one has

(1) $\psi \in \mathscr{L}^{1}\left(\lambda_{\zeta}\right)$ for $\nu$-a.e. $\zeta \in Y$,

(2) the function $\zeta \in Y \mapsto \int \psi(z) d \lambda_{\zeta}(z) \in \mathbf{C}$ determines an element of $L^{1}(\nu)$, and

(3) $\int \psi(z) d \mu(z)=\int\left\{\int \psi(z) d \lambda_{\zeta}(z)\right\} d \nu(\zeta)$.

A clean and elegant proof of the following result is set forth in the second section of $[\mathrm{AK}]$. Note where the measures of any disintegration must be concentrated!

Theorem. Suppose $X, \mu$, and $\varphi$ are as mentioned. Then there exists a disintegration $\zeta \in Y \mapsto \lambda_{\zeta} \in \mathscr{P}(X)$ of $\mu$ with respect to $\varphi$ such that $\lambda_{\zeta}$ is concentrated on $\varphi^{-1}(\zeta)$ for $\nu$-a.e. $\zeta \in Y$. Also, if $\zeta \in Y \mapsto \lambda_{\zeta}^{\prime} \in \mathscr{P}(X)$ is another disintegration of $\mu$ with respect to $\varphi$, then $\lambda_{\zeta}^{\prime}=\lambda_{\zeta}$ for $\nu$-a.e. $\zeta \in Y$.

One can easily see the following three facts from the definition of the measure $\nu$ and the definition of the disintegration $\zeta \in Y \mapsto \lambda_{\zeta} \in \mathscr{P}(X)$. 
Proposition. (i) If $\mu\left(\varphi^{-1}(\zeta)\right)=0$, then $\nu(\{\zeta\})=0$.

(j) If $\mu\left(\varphi^{-1}(\zeta)\right)>0$, then $\nu(\{\zeta\})>0$.

(k) If $\mu\left(\varphi^{-1}(\zeta)\right)>0$, then $\lambda_{\zeta}=\left[1 / \mu\left(\varphi^{-1}(\zeta)\right)\right] \cdot \mu \mid \varphi^{-1}(\zeta)$.

\section{THE MAIN RESULT}

For the duration of this section, $K, \mu$, and $f$ shall be fixed as in the introduction. Arbitrarily select a Borel representative from the equivalence class of $f \in L^{\infty}(\mu)$ and designate it too by $f$. Thus, $f$ is being viewed as an element of $\mathscr{L}^{\infty}(\mu)$. Taking the $X, \mu$, and $\varphi$ of the previous section to be the $K$, $\mu$, and $f$ of this section, get a disintegration $\zeta \in Y \mapsto \lambda_{\zeta} \in \mathscr{P}(K)$ of $\mu$ with respect to $f$.

Suppose $g^{\prime}$ and $g^{\prime \prime} \in \mathscr{L}^{\infty}(\mu)$ represent the same element of $L^{\infty}(\mu)$. Then $g^{\prime}=g^{\prime \prime} \quad \mu$-a.e. and so by setting $\psi=\left|g^{\prime}-g^{\prime \prime}\right|$ in (3) of the last section, one sees that $g^{\prime}=g^{\prime \prime} \lambda_{\zeta}$-a.e. for $\nu$-a.e. $\zeta \in Y$, i.e., $g^{\prime}$ and $g^{\prime \prime} \in \mathscr{L}^{\infty}\left(\lambda_{\zeta}\right)$ represent the same element of $L^{\infty}\left(\lambda_{\zeta}\right)$ for $\nu$-a.e. $\zeta \in Y$. Because of this, one may properly construe the phrase " $g \in R^{\infty}\left(E, \lambda_{\zeta}\right)$ for $\nu$-a.e. $\zeta \in Y$ " of the lemma that follows to mean that $g^{\prime}$ represents an element of $R^{\infty}\left(E, \lambda_{\zeta}\right)$ for $\nu$-a.e. $\zeta \in Y$ where $g^{\prime} \in \mathscr{L}^{\infty}(\mu)$ is any arbitrarily chosen representative of $g \in L^{\infty}(\mu)$. In what follows, for the sake of notational simplicity and because no harm thereby results, such careful distinctions between elements of $L^{\infty}(\mu)$ and their corresponding representatives in $\mathscr{L}^{\infty}(\mu)$ shall be left tacit.

Lemma. $A^{\infty}(f, \mu)=\left\{g \in L^{\infty}(\mu): g \in R^{\infty}\left(E, \lambda_{\zeta}\right)\right.$ for $\nu$-a.e. $\left.\zeta \in Y\right\}$.

Proof. Denote the right-hand side of the lemma's equality by $\mathscr{A}^{\infty}(f, \mu)$. Since $R(K) \subseteq R(E), R(K) \subseteq \mathscr{A}^{\infty}(f, \mu)$. Since $\lambda_{\zeta}$ is concentrated on $f^{-1}(\zeta)$ for $\nu$-a.e. $\zeta \in Y, f$ is constant as an element of $L^{\infty}\left(\lambda_{\zeta}\right)$ for $\nu$-a.e. $\zeta \in Y$ and so $\bar{f} \in \mathscr{A}^{\infty}(f, \mu)$. Clearly $\mathscr{A}^{\infty}(f, \mu)$, being an algebra, must then contain the algebra generated by $R(K)$ and $\bar{f}$. But the weak-star closure in $L^{\infty}(\mu)$ of the algebra generated by $R(K)$ and $\bar{f}$ is just $A^{\infty}(f, \mu)$. Hence to get $A^{\infty}(f, \mu) \subseteq \mathscr{A}^{\infty}(f, \mu)$, it suffices to show $\mathscr{A}^{\infty}(f, \mu)$ weak-star closed in $L^{\infty}(\mu)$. By the Krein-Schmulian Theorem, it in turn suffices to show ball $\mathscr{A}^{\infty}(f, \mu)$ weak-star closed in $L^{\infty}(\mu)$.

To this end, let $g$ be in the weak-star closure in $L^{\infty}(\mu)$ of ball $\mathscr{A}^{\infty}(f, \mu)$. Since $L^{2}(\mu) \subseteq L^{1}(\mu), g$ is in the weak closure in $L^{2}(\mu)$ of ball $\mathscr{A}^{\infty}(f, \mu)$. But the weak and strong closures of a convex subset of a Banach space coincide, so $g$ is in the strong closure in $L^{2}(\mu)$ of ball $\mathscr{A}^{\infty}(f, \mu)$. Accordingly, take $\left\{g_{n}\right\}_{n \geq 1}$ from ball $\mathscr{A}^{\infty}(f, \mu)$ such that $g_{n} \rightarrow g$ in $L^{2}(\mu)$. Passing to a subsequence, one may also assume that $g_{n} \rightarrow g \mu$-a.e.

Now select a Borel subset $\Delta$ of $K$ so that

(i) $\mu$ is concentrated on $\Delta$,

(ii) $\left|g_{n}\right| \leq 1$ on $\Delta$ for $n \geq 1$, and

(iii) $g_{n} \rightarrow g$ pointwise on $\Delta$. 
Setting $\psi=\chi_{K \backslash \Delta}$ in (3) of the last section and using (i), one sees that

(iv) $\lambda_{\zeta}$ is concentrated on $\Delta$ for $\nu$-a.e. $\zeta \in Y$.

Because of (ii), (iii), and (iv), the Lebesgue Dominated Convergence Theorem implies that $g_{n} \rightarrow g$ weak-star in $L^{\infty}\left(\lambda_{\zeta}\right)$ for $\nu$-a.e. $\zeta \in Y$. Since $\left\{g_{n}\right\}_{n \geq 1} \subseteq$ $\mathscr{A}^{\infty}(f, \mu),\left\{g_{n}\right\}_{n \geq 1} \subseteq R^{\infty}\left(E, \lambda_{\zeta}\right)$ for $\nu$-a.e. $\zeta \in Y$. Clearly then, $g \in$ $R^{\infty}\left(E, \lambda_{\zeta}\right)$ for $\nu$-a.e. $\zeta \in Y$ and so $g \in$ ball $\mathscr{A}^{\infty}(f, \mu)$. Thus, ball $\mathscr{A}^{\infty}(f, \mu)$ is weak-star closed in $L^{\infty}(\mu)$.

Having just established the inclusion $A^{\infty}(f, \mu) \subseteq \mathscr{A}^{\infty}(f, \mu)$, to obtain equality it suffices, by the Hahn-Banach Theorem, to show that $\mathscr{A}^{\infty}(f, \mu)$ is annihilated by any weak-star continuous annihilator of $A^{\infty}(f, \mu)$.

To this end, let $h \in L^{1}(\mu)$ annihilate $A^{\infty}(f, \mu)$. Choose $\left\{g_{n}\right\}_{n \geq 1}$ uniformly dense in $R(E)$. Suppose $p(\zeta, \bar{\zeta})$ is a polynomial in $\zeta$ and $\bar{\zeta}$. By Proposition (e), $p(f, \bar{f}) g_{n} \in A^{\infty}(f, \mu)$. Hence setting $\psi=p(f, \bar{f}) g_{n} h$ in (3) of the last section, one has

$$
\begin{aligned}
0 & =\int p(f, \bar{f}) g_{n} h d \mu \\
& =\int\left\{\int p(f(z), \overline{f(z)}) g_{n}(z) h(z) d \lambda_{\zeta}(z)\right\} d \nu(\zeta) \\
& =\int p(\zeta, \bar{\zeta})\left\{\int g_{n}(z) h(z) d \lambda_{\zeta}(z)\right\} d \nu(\zeta)
\end{aligned}
$$

Here the last equality follows since $\lambda_{\zeta}$ is concentrated on $f^{-1}(\zeta)$ for $\nu$-a.e. $\zeta \in Y$, thus making $p(f(z), \overline{f(z)})=p(\zeta, \bar{\zeta})$ for $\lambda_{\zeta}$-a.e. $z$ for $\nu$-a.e. $\zeta \in Y$. By the Stone-Weierstrass Theorem, the $L^{1}(\nu)$ function $\zeta \in Y \mapsto \int g_{n} h d \lambda_{\zeta} \in \mathbf{C}$ annihilates all continuous functions on $Y$. Hence $\int g_{n} h d \lambda_{\zeta}=0$ for $\nu-$ a.e. $\zeta \in Y$. But $n \geq 1$ is arbitrary and $\left\{g_{n}\right\}_{n \geq 1}$ is weak-star dense in $R^{\infty}\left(E, \lambda_{\zeta}\right)$ for every $\zeta \in Y$, so it follows that $h \in L^{1}\left(\lambda_{\zeta}\right)$ annihilates $R^{\infty}\left(E, \lambda_{\zeta}\right)$ for $\nu$-a.e. $\zeta \in Y$.

Now take $g \in \mathscr{A}^{\infty}(f, \mu)$. Then, by definition, $g \in R^{\infty}\left(E, \lambda_{\zeta}\right)$ for $\nu$-a.e. $\zeta \in Y$. Thus, setting $\psi=g h$ in (3) of the last section, one sees that

$$
\int g h d \mu=\int\left\{\int g(z) h(z) d \lambda_{\zeta}(z)\right\} d \nu(\zeta)=0 .
$$

Thus, $h \in L^{1}(\mu)$ annihilates $\mathscr{A}^{\infty}(f, \mu)$ and so $\mathscr{A}^{\infty}(f, \mu)=A^{\infty}(f, \mu)$.

Lemma. $R^{\infty}\left(E, \lambda_{\zeta}\right)=R^{\infty}\left(\check{f}^{-1}(\zeta), \lambda_{\zeta}\right)$ for $\nu$-a.e. $\zeta \in Y$.

Proof. By Proposition (g), there exists a sequence $\left\{f_{n}\right\}_{n \geq 1}$ from $R(E)$ such that

$$
f_{n} \rightarrow f \quad \text { boundedly } \mu \text {-a.e. }
$$

Let $\Delta$ be a Borel subset of $K$ on which $\mu$ is concentrated such that $f_{n} \rightarrow f$ pointwise boundedly on $\Delta$. Setting $\psi=\chi_{K \backslash \Delta}$ in (3) of the last section, one 
sees that $\lambda_{\zeta}$ is concentrated on $\Delta$ for $\nu$-a.e. $\zeta \in Y$. Thus $f_{n} \rightarrow f$ boundedly $\lambda_{\zeta}$-a.e. for $\nu$-a.e. $\zeta \in Y$. Since $\lambda_{\zeta}$ is concentrated on $f^{-1}(\zeta)$ for $\nu$-a.e. $\zeta \in Y$; it follows that for $\nu$-a.e. $\zeta \in Y$,

$$
f_{n} \rightarrow \zeta \quad \text { boundedly } \lambda_{\zeta} \text {-a.e. }
$$

Consequently, it suffices to show $R^{\infty}\left(E, \lambda_{\zeta}\right)=R^{\infty}\left(\check{f}^{-1}(\zeta), \lambda_{\zeta}\right)$ for any $\zeta \in Y$ such that (ii) holds.

Since $\check{f}^{-1}(\zeta) \subseteq E, R^{\infty}\left(E, \lambda_{\zeta}\right) \subseteq R^{\infty}\left(\check{f}^{-1}(\zeta), \lambda_{\zeta}\right)$. Thus, to obtain equality it suffices, by the Hahn-Banach Theorem, to show that $R^{\infty}\left(\check{f}^{-1}(\zeta), \lambda_{\zeta}\right)$ is annihilated by any weak-star continuous annihilator of $R^{\infty}\left(E, \lambda_{\zeta}\right)$.

To this end, let $h \in L^{1}\left(\lambda_{\zeta}\right)$ annihilate $R^{\infty}\left(E, \lambda_{\zeta}\right)$ and consider the measure $\sigma$ defined by $d \sigma=h d \lambda_{\zeta}$. By hypothesis, $\sigma$ annihilates $R(E)$, i.e., $\hat{\sigma}=0$ $A$-a.e. off $E$. Since $\sigma<<\lambda_{\zeta}$, to prove that $h$ annihilates $R^{\infty}\left(\check{f}^{-1}(\zeta), \lambda_{\zeta}\right)$ it suffices to show that $\sigma$ annihilates $R\left(\breve{f}^{-1}(\zeta)\right)$, i.e., $\hat{\sigma}=0 \quad A$-a.e. off $\check{f}^{-1}(\zeta)$. Let $\tilde{\sigma}\left(z_{0}\right)=\int \frac{d|\sigma|(z)}{\left|z-z_{0}\right|}$ be the Newtonian potential of $\sigma$. Since $\tilde{\sigma}<\infty \quad A$-a.e. on $\mathbf{C}$, it in turn suffices to show $\hat{\sigma}\left(z_{0}\right)=0$ whenever

$$
z_{0} \in E \backslash \check{f}^{-1}(\zeta) \quad \text { and } \quad \tilde{\sigma}\left(z_{0}\right)<\infty .
$$

By Proposition (c), there exists a sequence $\left\{g_{n}\right\}_{n \geq 1}$ from $R(E)$, with each $g_{n}$ extending to be holomorphic near $z_{0}$, such that

$$
\left\|g_{n}-f_{n}\right\|_{K} \rightarrow 0 \text {. }
$$

By (i) and (iv), $g_{n} \rightarrow f$ boundedly $\mu$-a.e. and so $\check{g}_{n} \rightarrow \check{f}$ pointwise boundedly on $E$. But then by (iii) and Proposition (f),

$$
g_{n}\left(z_{0}\right)=\check{g}_{n}\left(z_{0}\right) \rightarrow \check{f}\left(z_{0}\right) .
$$

Since $\sigma<<\lambda_{\zeta}$, by (ii) and (iv) one has

$$
g_{n} \rightarrow \zeta \quad \text { boundedly } \sigma \text {-a.e. }
$$

Because of (iii), (v), and (vi), the Lebesgue Dominated Convergence Theorem implies that

$$
\lim _{n \rightarrow \infty} \int \frac{g_{n}(z)-g_{n}\left(z_{0}\right)}{z-z_{0}} d \sigma(z)=\int \frac{\zeta-\check{f}\left(z_{0}\right)}{z-z_{0}} d \sigma(z) .
$$

The left-hand side of (vii) is zero since $\sigma$ annihilates $R(E)$ and each $\left(g_{n}(z)-\right.$ $\left.g_{n}\left(z_{0}\right)\right) /\left(z-z_{0}\right) \in R(E)$ by Proposition (b). Since $\zeta-\check{f}\left(z_{0}\right)$ is constant as far as the integration is concerned, the right-hand side of (vii) is just $\left\{\zeta-\check{f}\left(z_{0}\right)\right\} \hat{\sigma}\left(z_{0}\right)$. Thus, $\left\{\zeta-\check{f}\left(z_{0}\right)\right\} \hat{\sigma}\left(z_{0}\right)=0$. By (iii), $\zeta-\check{f}\left(z_{0}\right) \neq 0$, so it must be that $\hat{\sigma}\left(z_{0}\right)=$ 0 .

Let $\widetilde{a_{1}}, \widetilde{a_{2}}, \cdots$ be an enumeration of the at most countable set of points $\zeta \in \mathbf{C}$ such that $\mu\left(f^{-1}(\zeta)\right)>0$. Set $\tilde{\mu}_{i}=\mu \mid f^{-1}\left(\tilde{a}_{i}\right)$ and $\tilde{\mu}_{s}=\mu-\sum \tilde{\mu}_{i}$. The 
main result of [GRT] states that $A^{\infty}(f, \mu)=L^{\infty}\left(\mu_{s}\right) \oplus \sum \oplus R^{\infty}\left(K, \tilde{\mu}_{i}\right)$ where the right-hand side of the equality is interpreted to mean $\left\{g \in L^{\infty}(\mu): g \in\right.$ $R^{\infty}\left(K, \tilde{\mu}_{i}\right)$ for each $\left.i=1,2, \ldots\right\}$. Compare this to the main result of the present paper, which follows immediately.

Theorem. Let $a_{1}, a_{2}, \ldots$ be an enumeration of the at most countable set of points $\zeta \in \mathbf{C}$ such that both $\mu\left(f^{-1}(\zeta)\right)>0$ and $A\left(\check{f}^{-1}(\zeta)\right)>0$. Set $\mu_{i}=\mu \mid f^{-1}\left(a_{i}\right)$ and $\mu_{s}=\mu-\sum \mu_{i}$. Then

$$
A^{\infty}(f, \mu)=L^{\infty}\left(\mu_{s}\right) \oplus \sum \oplus R^{\infty}\left(\check{f}^{-1}\left(a_{i}\right), \mu_{i}\right) .
$$

Proof. Set $\Omega=\left\{\zeta \in \mathbf{C}: \mu\left(f^{-1}(\zeta)\right)>0\right.$ and $\left.A\left(\check{f}^{-1}(\zeta)\right)>0\right\}$ and $\Omega^{\prime}=\{\zeta \in \mathbf{C}$ : $\mu\left(f^{-1}(\zeta)\right)=0$ and $\left.A\left(\check{f}^{-1}(\zeta)\right)>0\right\}$. Note that both $\Omega$ and $\Omega^{\prime}$ are countable with $\Omega=\left\{a_{1}, a_{2}, \ldots\right\}$ and $\Omega \cup \Omega^{\prime}=\left\{\zeta \in \mathbf{C}: A\left(\check{f}^{-1}(\zeta)\right)>0\right\}$. Then

$$
A^{\infty}(f, \mu)=\left\{g \in L^{\infty}(\mu): g \in R^{\infty}\left(E, \lambda_{\zeta}\right) \text { for } \nu \text {-a.e. } \zeta \in Y\right\}
$$

(by Lemma One)

$$
\begin{aligned}
& =\left\{g \in L^{\infty}(\mu): g \in R^{\infty}\left(\check{f}^{-1}(\zeta), \lambda_{\zeta}\right) \text { for } \nu \text {-a.e. } \zeta \in Y\right\} \\
& =\left\{g \in L^{\infty}(\mu): g \in R^{\infty}\left(\check{f}^{-1}(\zeta), \lambda_{\zeta}\right) \text { for } \nu \text {-a.e. } \zeta \in \Omega \cup \Omega^{\prime}\right\} \\
& =\left\{g \in L^{\infty}(\mu): g \in R^{\infty}\left(\check{f}^{-1}(\zeta), \lambda_{\zeta}\right) \text { for } \nu \text {-a.e. } \zeta \in \Omega\right\} \\
& =\left\{g \in L^{\infty}(\mu): g \in R^{\infty}\left(\check{f}^{-1}(\zeta), \lambda_{\zeta}\right) \text { for every } \zeta \in \Omega\right\} \\
& =\left\{g \in L^{\infty}(\mu): g \in R^{\infty}\left(\check{f}^{-1}(\zeta), \mu \mid f^{-1}(\zeta)\right) \text { for every } \zeta \in \Omega\right\} \\
& \quad \text { (by Proposition (i) }) \\
& =\left\{g \in L^{\infty}(\mu): g \in R^{\infty}\left(\check{f}^{-1}\left(a_{i}\right), \mu_{i}\right) \text { for each } i=1,2, \ldots\right\} \\
& =L^{\infty}\left(\mu_{s}\right) \oplus \sum \oplus R^{\infty}\left(\check{f}^{-1}\left(a_{i}\right), \mu_{i}\right) .
\end{aligned}
$$$$
\text { (by Proposition }(\mathrm{h}) \text { ) }
$$$$
\text { (by Proposition }(\mathrm{j}) \text { ) }
$$

Of course there may be no $a_{i}$ 's, in which case one has the following:

Corollary. $A^{\infty}(f, \mu)=L^{\infty}(\mu)$ whenever each $\zeta \in \mathbf{C}$ is such that either

$$
\mu\left(f^{-1}(\zeta)\right)=0 \text { or } A\left(\check{f}^{-1}(\zeta)\right)=0 .
$$

A necessary and sufficient condition for weak-star density is contained in the following:

\section{Corollary.}

$$
A^{\infty}(f, \mu)=L^{\infty}(\mu) \quad \text { iff } R^{\infty}\left(\check{f}^{-1}(\zeta), \mu \mid f^{-1}(\zeta)\right)=L^{\infty}\left(\mu \mid f^{-1}(\zeta)\right)
$$

for each $\zeta \in \mathbf{C}$. 


\section{ACKNOWLEDGMENTS}

The author would like to express his thanks to John B. Conway for many helpful conversations in connection with this work.

\section{REFERENCES}

[AK] M. B. Abrahamse and T. L. Kriete, The spectral multiplicity of a multiplication operator, Indiana Univ. Math. J. 22 (1973), 845-857.

[Ch] J. Chaumat, Adherence faible étoile d'algebres de fractions rationelles, Publications Mathématique d'Orsay, No. 147.

[C] J. B. Conway, 'The minimal normal extension of a function of a subnormal operater' in Proceedings of the Special Year in Operator Theory at the University of Illinois, Cambridge Univ. Press, Cambridge (to appear).

[CO] J. B. Conway and R. F. Olin, A functional calculus for subnormal operators, II, Memoirs Amer. Math. Soc. No. 184, Providence, R.I.

[D] J. J. Dudziak, The minimal normal extension problem for subnormal operators, J. Funct. Anal. 65 (1986), 314-338.

[G1] T. W. Gamelin, Uniform algebras, Prentice Hall, Englewood Cliffs, N.J.

[G2] _ Rational approximation theory, Univ. California at Los Angeles Lecture Notes (unpublished), Los Angeles, CA.

[GRT] T. W. Gamelin, P. Russo and J. E. Thomson, A Stone-Weierstrass theorem for weak-star approximation by rational functions, J. Funct. Anal. (to appear).

Mathematics Department, Bucknell University, Lewisburg, Pennsylvania 17837 